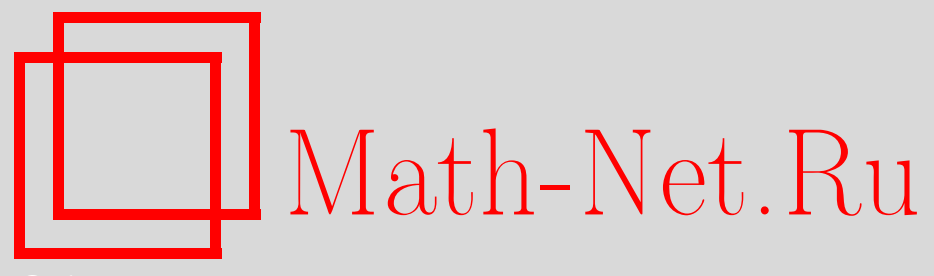

Х. Д. Икрамов, Квадратично нормальные матрицы типа 1 и унитарные подобия между ними, Матем. заметки, 2009, том 86, выпуск 3, 371-379

DOI: https://doi.org/10.4213/mzm8500

Использование Общероссийского математического портала Math-Net.Ru подразумевает, что вы прочитали и согласны с пользовательским соглашением http://www . mathnet.ru/rus/agreement

Параметры загрузки:

IP : 3.80 .181 .102

26 апреля 2023 г., 09:24:35

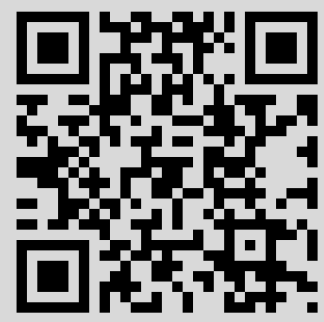


Том 86 выпуск 3 сентябрь 2009

УДК 517

\section{Квадратично нормальные матрицы типа 1 и унитарные подобия между ними}

\section{Х. Д. Икрамов}

Известно, что проверка унитарного подобия между матрицами, имеющими квадратичные минимальные многочлены, требует многократно меньшей вычислительной работы, чем использование общего критерия Шпехта-Пирси. Возможность такой экономии вычислений опирается на следующий факт: если $(n \times n)$-матрицы $A$ и $B$ имеют квадратичные минимальные многочлены, то для унитарного подобия между ними необходимо и достаточно, чтобы $A$ и $B$ имели одинаковые собственные значения и одинаковые сингулярные числа. Показано, что этот факт справедлив и для некоторого подкласса матриц с кубическими минимальными многочленами, а именно для квадратично нормальных матриц типа 1.

Библиография: 7 названий.

1. Введение. Проверка унитарного подобия между комплексными $(n \times n)$-матрицами $A$ и $B$ теоретически может быть проведена посредством классической теоремы Шпехта (см. [1; теорема 2.2.6]). Обозначим через $M_{n}$ множество матриц порядка $n$ над $\mathbb{C}$.

Теорема 1 (Шпехт). Матрицы $A, B \in M_{n}$ унитарно подобнъ тогда и только тогда, когда

$$
\operatorname{tr} W\left(A, A^{*}\right)=\operatorname{tr} W\left(B, B^{*}\right)
$$

для любого слова

$$
W(s, t)=s^{m_{1}} t^{n_{1}} \ldots s^{m_{k}} t^{n_{k}}, \quad m_{1}, n_{1}, \ldots, m_{k}, n_{k} \geqslant 0,
$$

от некоммутирующих переменных $s$ и

Теорема 1 не является эффективным критерием, поскольку требует проверки бесконечного числа условий (1). Однако она была превращена в эффективный критерий в статье [2] (см. также [1; теорема 2.2.8]). Назовем число

$$
m_{1}+n_{1}+\cdots+m_{k}+n_{k}
$$

длиной слова (2).

Теорема 2 (Пирси). Матрицы $A, B \in M_{n}$ унитарно подобны тогда и толъко тогда, когда равенства (1) выполняются для всех слов $W(s, t)$, длина которых не nревосходит $2 n^{2}$.

(C) Х. Д. Икрамов, 2009 
Итак, наличие или отсутствие унитарного подобия между $A$ и $B$ может быть проверено посредством конечного вычисления. Однако применение критерия Шпехта-Пирси к матрицам общего вида требует огромной вычислительной работы. Информация о принадлежности $A$ и $B$ специальным матричным классам в некоторых случаях позволяет многократно сократить эту работу. Так, для унитарного подобия нормальных матриц $A, B \in M_{n}$ необходимо и достаточно, чтобы эти матрицы имели одинаковые собственные значения. Проверка последнего условия сводится к проверке $n$ равенств

$$
\operatorname{tr}\left(A^{i}\right)=\operatorname{tr}\left(B^{i}\right), \quad i=1, \ldots, n,
$$

что является впечатляющей экономией вычислительной работы по сравнению с теоремой 2 .

$\mathrm{K}$ тематике настоящей статьи ближе случай, когда об $A$ и $B$ известно, что обе матрицы имеют квадратичные минимальные многочлены. Этот случай был рассмотрен в [3].

Теорема 3. Если матрицы $A, B \in M_{n}$ имеют квадратичные минимальные многочлены, то для унитарного подобия между ними необходимо и достаточно, чтобы А и В имели одинаковые собственные значения и одинаковые сингулярные числа.

Проверка условий теоремы 3 включает в себя наряду с (3) проверку $n$ равенств

$$
\operatorname{tr}\left(A A^{*}\right)^{i}=\operatorname{tr}\left(B B^{*}\right)^{i}, \quad i=1, \ldots, n .
$$

Однако, как замечено Альпиным (см. [4]), число проверяемых следовых равенств можно сократить с $2 n$ до $[n / 2]+3$.

Цель данной статьи - распространить утверждение теоремы 3 на некоторый подкласс матриц с кубическими минимальными многочленами. Назовем матрицу $A \in M_{n}$ полиномиально нормальной степени $d$, если существует унитарный многочлен $p(z)$ степени $d$ такой, что матрица $p(A)$ нормальна, и $q(A)$ не является нормальной матрицей ни для какого многочлена $q(z)$ степени меньшей, чем $d$. (Это определение заимствовано из [5], [6].) В этой терминологии обычные нормальные матрицы суть полиномиально нормальные матрицы степени 1.

Нас будут интересовать полиномиально нормальные матрицы степени 2 или, короче говоря, квадратично нормалъные матрицы. Унитарный многочлен $p(z)$ в определении такой матрицы будем называть ее нормальным многочленом. Мы скажем, что квадратично нормальная матрица $A$ имеет тип $k$, если ранг нормальной матрицы $p(A)$ равен $k$. В частности, матрицы с квадратичными минимальными многочленами являются квадратично нормальными матрицами нулевого типа.

В настоящей статье мы изучаем квадратично нормальные матрицы типа 1. В п. 2 исследована структура матриц этого класса. Опираясь на это исследование, мы устанавливаем в п. 3 основной результат статьи:

Теорема 4. Если $A, B \in M_{n}$ - квадратично нормалъные матрицы типа 1, то для унитарного подобия между ними необходимо и достаточно, чтобъ $A$ u $B$ имели одинаковые собственные значения и одинаковые сингулярные числа.

В заключительном пункте мы обсуждаем вопрос о том, как проверить посредством конечного вычисления, является ли заданная матрица $A \in M_{n}$ квадратично нормальной типа 1. 
2. Структура матриц. Множество квадратично нормальных $(n \times n)$-матриц типа 1 будем обозначать символом $\mathscr{Q} \mathscr{N}_{n}^{1}$. Заметим, что это множество непусто лишь при $n \geqslant 3$. Пусть $A \in \mathscr{Q}_{n}^{1}$ и

$$
p(z)=z^{2}+\alpha z+\beta
$$

есть нормальный многочлен матрицы $A$. Положим

$$
B=p(A)=A^{2}+\alpha A+\beta I_{n} .
$$

По условию нормальная матрица $B$ имеет ранг 1 . Следовательно, $B$ имеет ядро $\mathscr{Z}$ размерности $n-1$ и одномерный образ $\mathscr{R}$. Последний можно рассматривать как одномерное инвариантное подпространство матрицы $B$, отвечающее ее единственному ненулевому собственному значению $b=\operatorname{tr} B$.

Поскольку $B$ есть многочлен от $A$, матрицы $A$ и $B$ перестановочны:

$$
A B=B A .
$$

Отсюда следует, что подпространства $\mathscr{R}$ и $\mathscr{Z}$ инвариантны относительно $A$.

Обозначим через $A_{2}$ сужение матрицы $A$ на подпространство $\mathscr{Z}$. Поскольку сужение $B$ на $\mathscr{Z}$ есть нулевая матрица, из (6) следует

$$
p\left(A_{2}\right)=A_{2}^{2}+\alpha A_{2}+\beta I_{n-1}=0,
$$

т.е. многочлен $p(z)$ аннулирует $A_{2}$. В действительности, как мы сейчас увидим, $p(z)$ есть минимальный многочлен этой матрицы.

Одномерное подпространство $\mathscr{R}$ является для $A$ собственным подпространством. Пусть $a$ - собственное значение, ассоциированное с $\mathscr{R}$. Тогда

$$
b=p(a)=a^{2}+\alpha a+\beta .
$$

Как отмечено выше, $b \neq 0$. Если бы $A_{2}$ аннулировалась линейным многочленом $z-\lambda_{0}$ (т.е. была бы скалярной матрицей $\lambda_{0} I_{n-1}$ ), то квадратный трехчлен $q(z)=$ $\left(z-\lambda_{0}\right)(z-a)$ был бы минимальным многочленом для $A$, и $A$ была бы квадратично нормальной матрицей нулевого типа, а не типа 1.

Условимся называть $а$ особенным собственным значением матрицы $A$. От прочих собственных значений этой матрицы оно отличается тем, что

$$
p(a) \neq 0 .
$$

Первым следствием проведенного анализа является

ПреДЛОЖение 1. Всякая матрица $A \in \mathscr{Q}_{n}^{1}$ имеет кубический минимальный многочлен.

В самом деле, $A$ не может быть аннулирована никаким многочленом второй степени; в то же время $A$ аннулируется многочленом

$$
r(z)=(z-a) p(z)
$$

Для дальнейших выводов нам потребуется следующий результат из [7] (см. также [4]). 
Теорема 5. Пусть $A \in M_{n}$ - матрица с квадратичным минимальным многочленом. Если А имеет единственное собственное значение $\mu$, то подходящим унитарным подобием $A$ может быть приведена к виду

$$
\left(\begin{array}{cc}
\mu & \sigma_{1} \\
0 & \mu
\end{array}\right) \oplus \cdots \oplus\left(\begin{array}{cc}
\mu & \sigma_{k} \\
0 & \mu
\end{array}\right) \oplus \mu \oplus \cdots \oplus \mu .
$$

При наличии двух различных собственных значений $\mu$ и существует унитарное подобие, приводящее $A \kappa$ виду

$$
\left(\begin{array}{cc}
\mu & \sigma_{1} \\
0 & \nu
\end{array}\right) \oplus \cdots \oplus\left(\begin{array}{cc}
\mu & \sigma_{k} \\
0 & \nu
\end{array}\right) \oplus \lambda_{1} \oplus \cdots \oplus \lambda_{l}
$$

¿əe

$$
2 k+l=n, \quad \lambda_{j} \in\{\mu, \nu\}, \quad 1 \leqslant j \leqslant l .
$$

Числа $\sigma_{1}, \ldots, \sigma_{k}$ в суммах (8) и (9) отличны от нуля и могут быть выбраны положительными.

Заметим, что в прямой сумме (8) присутствует хотя бы одно слагаемое порядка 2. В противном случае $A$ была бы скалярной матрицей $\mu I_{n}$. Если блоки порядка 2 отсутствуют в прямой сумме (9), то $A$ - нормальная матрица.

Соединяя проведенный выше анализ с теоремой 5 , приходим к следующему заключению.

ПреДЛОЖение 2. Всякая матрица $A \in \mathscr{Q}_{n}^{1}$ посредством подходящего унитарного подобия может быть преобразована в прямую сумму

$$
S=a \oplus S_{n-1} .
$$

Здесъ а-особенное собственное значение, а $S_{n-1}$ имеет вид прямой суммъ (8) или (9), где числа н и суть корни нормального многочлена матрицы $A$. В обоих случаях прямая сумма содержит хотя бы одно слагаемое порядка 2.

ДокАЗАтЕЛьство. В обосновании нуждается только последнее утверждение. Если допустить, что $S_{n-1}$ - скалярная матрица, то $A$ - матрица с квадратичным минимальным многочленом. Если же сумма (9) является диагональной матрицей, то $A$ нормальна. В обоих случаях получаем противоречие с условием $A \in \mathscr{Q} \mathscr{N}_{n}^{1}$.

3. Доказательство теоремы 4. Необходимость условий теоремы очевидна. Докажем, что матрицы $A, B \in \mathscr{Q} \mathscr{N}_{n}^{1}$, имеющие общий список $\mathscr{E}$ собственных значений и общий список $\mathscr{S}$ сингулярных чисел, должны быть унитарно подобны. Для этого мы покажем, что форма (11), к которой каждая из матриц $A$ и $B$ может быть приведена унитарным подобием, однозначно определяется двумя указанными списками.

Как следует из п. 2, список $\mathscr{E}$ может содержать лишь два или три различных числа. Исследуем эти возможности по отдельности.

Если в $\mathscr{E}$ встречаются только два числа, то одно из них простое и является особенным собственным значением, а другое обязано иметь кратность $n-1$. Обозначим эти числа соответственно $a$ и $\mu$. Матрица $S_{n-1}$ должна иметь форму (8), где присутствует хотя бы один блок порядка 2 , а число $k$ таких блоков еще предстоит определить. 
Заметим, что квадраты сингулярных чисел матрицы

$$
\left(\begin{array}{cc}
\mu & \sigma \\
0 & \mu
\end{array}\right), \quad \sigma>0
$$

даются формулой

$$
z_{1,2}=|\mu|^{2}+\frac{1}{2}\left\{\sigma^{2} \pm \sqrt{\sigma^{4}+4|\mu|^{2} \sigma^{2}}\right\}
$$

Если $\mu \neq 0$, то одно из чисел $z$ строго больше, а другое строго меньше, чем $|\mu|^{2}$. Поэтому, если исключить из списка $\mathscr{S}$ число $|a|$, соответствующее особенному собственному значению, то число пар в оставшемся списке, имеющих вид

$$
(\widetilde{s}, \widehat{s}), \quad \text { где } \quad \widetilde{s}>|\mu|, \quad \widehat{s}<|\mu|,
$$

указывает число $k$ блоков 2-го порядка в матрице $S_{n-1}$. Теперь $S_{n-1}$ определена полностью.

Рассмотрим теперь случай, когда $\mathscr{E}$ содержит три различных числа. Если лишь одно из них простое, то оно и является особенным собственным значением $a$. K разбору этой ситуации мы вернемся позже. Однако может случиться, что в $\mathscr{E}$ присутствуют два простых собственных значения. Одно из них - особенное собственное значение $a$, а другое (назовем его $\nu$ ) относится к $S_{n-1}$ и обязательно входит в клетку 2-го порядка

$$
\left(\begin{array}{cc}
\mu & \sigma \\
0 & \nu
\end{array}\right), \quad \sigma>0
$$

Спрашивается, как отличить $a$ от $\nu$ ?

Квадраты сингулярных чисел матрицы (14) даются формулой

$$
z_{1,2}=\frac{|\mu|^{2}+|\nu|^{2}}{2}+\frac{1}{2}\left\{\sigma^{2} \pm\left[\left(|\mu|^{2}-|\nu|^{2}\right)^{2}+2\left(|\mu|^{2}+|\nu|^{2}\right) \sigma^{2}+\sigma^{4}\right]^{1 / 2}\right\} .
$$

Если $\mu \nu \neq 0$, то одно из чисел $z$ строго больше наибольшего из чисел $|\mu|^{2}$ и $|\nu|^{2}$, а другое строго меньше, чем $\min \left\{|\mu|^{2},|\nu|^{2}\right\}$. Отсюда следует, что список $\mathscr{S}$ не содержит числа $|\nu|$; в то же время, он должен содержать число $|a|$. Итак, число $a$ отличается от $\nu$ тем, что его модуль входит в $\mathscr{S}$. Сам список $\mathscr{S}$ выглядит так:

$$
\mathscr{S}=\left\{|a|, \sqrt{z_{1}}, \sqrt{z_{2}},|\mu|, \ldots,|\mu|\right\} .
$$

Матрица $S_{n-1}$ в данном случае имеет вид

$$
\left(\begin{array}{cc}
\mu & \sigma \\
0 & \nu
\end{array}\right) \oplus \mu \oplus \cdots \oplus \mu
$$

Если $\mu=0$, то формула (15) дает

$$
z_{1}=|\nu|^{2}+\sigma^{2}, \quad z_{2}=0 .
$$

Следовательно,

$$
\mathscr{S}=\left\{|a|, \sqrt{z_{1}}, 0, \ldots, 0\right\} .
$$

Поскольку $\sqrt{z_{1}}>|\nu|$, число $a$ теперь выделяется следующим описанием: это ненулевое простое собственное значение, модуль которого входит в $\mathscr{S}$. Матрица $S_{n-1}$ по-прежнему имеет форму (16), где $\mu=0$. 
Пусть, наконец, $\nu=0$. Тогда из (15) получаем

$$
z_{1}=|\mu|^{2}+\sigma^{2}, \quad z_{2}=0 .
$$

Поэтому

$$
\mathscr{S}=\left\{|a|, \sqrt{z_{1}}, 0,|\mu|, \ldots,|\mu|\right\} .
$$

Описание особенного собственного значения, предложенное в предыдущем абзаце, сохраняет силу и в данном случае. Действительно, при $n=3$ число $\mu$ также является ненулевым и простым, но его модуль не входит в $\mathscr{S}$. Если же $n \geqslant 4$, то $\mu$ будет кратным собственным значением. Матрица $S_{n-1}$ имеет форму (16), где $\nu=0$.

Нам остается исследовать случай, когда $\mathscr{E}$ содержит лишь одно простое собственное значение. Это сразу определяет $a$, но точную форму $S_{n-1}$ (т.е. число $k$ блоков 2-го порядка) еще предстоит найти. Из нашего предыдущего анализа можно извлечь такое правило для определения $k$ : удалить $|a|$ из списка $\mathscr{S}$ и найти в оставшемся списке число пар вида

$$
(\widetilde{s}, \widehat{s}), \quad \text { где } \quad \widetilde{s}>\max \{|\mu|,|\nu|\}, \quad \widehat{s} \leqslant \min \{|\mu|,|\nu|\} .
$$

Если $\mu \nu \neq 0$, то в правом неравенстве знак $\leqslant$ следует заменить на $<$.

Количество пар типа (17) дает искомое число $k$. Матрица $S_{n-1}$ теперь определена полностью.

Мы разобрали все возможные случаи и в каждом из них показали, что форма (11) однозначно восстанавливается по спискам $\mathscr{E}$ и $\mathscr{S}$. Тем самым доказана достаточность условий теоремы 4.

4. Принадлежность классу $\mathscr{Q} \mathscr{N}_{n}^{1}$. В этом пункте мы обсудим вопрос о том, как проверить принадлежность заданной матрицы $A \in M_{n}$ классу $\mathscr{Q} \mathscr{N}_{n}^{1}$.

Теорема 6. Для всякой матрицы $A \in \mathscr{Q}_{n}^{1}$ нормалъный многочлен определен единственным образом.

ДокАЗАТЕЛЬСТво. Предположим, что

$$
p_{1}(z)=z^{2}+\alpha_{1} z+\beta_{1} \quad \text { и } \quad p_{2}(z)=z^{2}+\alpha_{2} z+\beta_{2}
$$

суть нормальные многочлены для $A$, т.е. матрицы

$$
\begin{aligned}
& B_{1}=A^{2}+\alpha_{1} A+\beta_{1} I_{n}, \\
& B_{2}=A^{2}+\alpha_{2} A+\beta_{2} I_{n}
\end{aligned}
$$

нормальны. Вычитая (19) из (18), имеем

$$
\left(\alpha_{1}-\alpha_{2}\right) A=B_{1}-B_{2}+\left(\beta_{2}-\beta_{1}\right) I_{n}
$$

Матрицы $B_{1}$ и $B_{2}$, будучи многочленами от одной и той же матрицы $A$, перестановочны. Разность перестановочных нормальных матриц $B_{1}$ и $B_{2}$ также является нормальной матрицей. Но тогда нормальна и правая часть равенства (20). Если допустить, что $\alpha_{1} \neq \alpha_{2}$, то из (20) будет следовать, что сама матрица $A$ нормальна, тогда как $A$ лишь квадратично нормальна. 
Итак, $\alpha_{1}=\alpha_{2}$. Перепишем равенство (20) в виде

$$
B_{2}=B_{1}+\left(\beta_{2}-\beta_{1}\right) I_{n}
$$

По условию, каждая из матриц $B_{1}$ и $B_{2}$ должна иметь собственное значение нуль кратности $n-1 \geqslant 2$. Однако, если это условие выполняется для $B_{1}$ и при этом $\beta_{1} \neq \beta_{2}$, то собственным значением кратности $n-1$ для $B_{2}$ является ненулевое число $\beta_{2}-\beta_{1}$, а не нуль. Поэтому $\beta_{1}=\beta_{2}$, что доказывает теорему.

ЗАмечАниЕ. Если не требовать, чтобы квадратично нормальная матрица $A$ имела определенный тип, то в выборе нормального многочлена имеется некоторая свобода. В самом деле, если матрица (6) нормальна, то для любого $\Delta \in \mathbb{C}$ нормальна и матрица

$$
B+\Delta I_{n}=A^{2}+\alpha A+(\beta+\Delta) I_{n},
$$

т.е., наряду с (5), нормальным является и многочлен

$$
p_{\Delta}(z)=z^{2}+\alpha z+(\beta+\Delta) .
$$

Однако коэффициент $\alpha$ и в этом случае определен единственным образом. Действительно, первая часть доказательства теоремы 6 использовала лишь нормальность матриц $B_{1}$ и $B_{2}$. Значения их рангов были при этом несущественны.

Единственность многочлена $p(z)$ можно сохранить, отказавшись от нормальности матрицы $p(A)$, но сохранив другое требование к $A$, а именно, чтобы $A$ имела кубический минимальный многочлен.

Теорема 7. Пусть матрица $A \in M_{n}$ имеет кубический минимальный многочлен. Если существует унитарный многочлен

$$
p(z)=z^{2}+\alpha z+\beta
$$

такой, что матрица

$$
B=p(A)=A^{2}+\alpha A+\beta I_{n}
$$

имеет ранг 1, то такой многочлен определен единственным образом.

ДокАЗАТЕЛЬСТво. Пусть подобие

$$
A \rightarrow J=Q^{-1} A Q
$$

приводит $A$ к ее жордановой форме $J$. Полагая

$$
C=Q^{-1} B Q
$$

заменим (21) равенством

$$
C=J^{2}+\alpha J+\beta I_{n}
$$

Матрица $A$ с кубическим минимальным многочленом имеет не более трех различных собственных значений. Если три различных собственных значения действительно имеются, обозначим их через $\lambda, \mu$ и $\nu$. Приравнивая нулю главные миноры 
порядка 2 в матрице $C$, убеждаемся, что, по крайней мере, для двух собственных значений, скажем, для $\lambda$ и $\mu$, должны выполняться равенства

$$
\begin{aligned}
& \lambda^{2}+\alpha \lambda+\beta=0, \\
& \mu^{2}+\alpha \mu+\beta=0 .
\end{aligned}
$$

Таким образом, $\lambda$ и $\mu$ суть корни искомого многочлена $p(z)$, откуда

$$
\alpha=-(\lambda+\mu), \quad \beta=\lambda \mu .
$$

Если $A$ имеет два различных собственных значения $\lambda$ и $\mu$ и по-прежнему выполнены равенства (23) и (24), то единственность $\alpha$ и $\beta$ доказывается прежним образом. Если выполнено, например, (24), но

$$
\lambda^{2}+\alpha \lambda+\beta \neq 0
$$

то $\lambda$ - простое собственное значение, а числу $\mu$ в жордановой форме $J$ соответствует хотя бы одна клетка порядка 2. Для определенности предположим, что $\lambda$ стоит в позиции $(1,1)$ матрицы $J$, а в ее позиции $(i, i+1), i \geqslant 2$, находится единица. Приравнивая нулю минор 2-го порядка матрицы $C$, опирающийся на строки 1 и $i$ и столбцы 1 и $i+1$, заключаем, что $\alpha=0$. Но тогда $\beta=-\mu^{2}$.

Если $\mu$-единственное собственное значение матрицы $A$, то $J$ должна содержать клетку порядка три. Пусть эта клетка располагается в строках и столбцах с индексами $i, i+1$ и $i+2$. Приравнивая нулю минор 2 -го порядка матрицы $C$, опирающийся на строки $i$ и $i+1$ и столбцы $i+1$ и $i+2$, снова приходим к равенству $\alpha=0$, откуда, как и выше, $\beta=-\mu^{2}$.

Опираясь на теоремы 6 и 7, можно предложить следующий способ проверки включения $A \in \mathscr{Q} \mathscr{N}_{n}^{1}$.

1. Решить относительно $\alpha$ и $\beta$ систему квадратичных уравнений

$$
f_{i}(\alpha, \beta)=0, \quad i=1,2, \ldots, N
$$

записывающих условие равенства нулю всех миноров 2-го порядка матрицы (21).

2. Если система (25) несовместна или совместна, но имеет неединственное решение, то включение $A \in \mathscr{Q} \mathscr{N}_{n}^{1}$ невозможно (см. теорему 7 и предложение 1 ). Пусть $\left(\alpha_{0}, \beta_{0}\right)$ - единственное решение системы $(25)$. В этом случае $A \in \mathscr{Q} \mathscr{N}_{n}^{1}$, если и только если матрица

$$
B_{0}=A^{2}+\alpha_{0} A+\beta_{0} I_{n}
$$

нормальна. Это условие можно проверить “в лоб", сравнивая матрицы $B_{0} B_{0}^{*}$ и $B_{0}^{*} B_{0}$, или же учесть то обстоятельство, что $B_{0}$ - матрица ранга 1. Если

$$
B_{0}=x y^{*}
$$

есть представление $B_{0}$ в виде диады, то для нормальности $B_{0}$ необходимо и достаточно, чтобы векторы $x$ и $y$ были коллинеарны:

$$
y=\gamma x, \quad \gamma \in \mathbb{C} .
$$




\section{СПИСОК ЦИТИРОВАННОЙ ЛИТЕРАТУРЫ}

[1] Р. Хорн, Ч. Джонсон, Матричный анализ, Мир, М., 1989.

[2] C. Pearcy, "A complete set of unitary invariants for operators generating finite $W^{*}$-algebras of type I", Pacific J. Math., 12 (1962), 1405-1416.

[3] A. George, Kh. D. Ikramov, "Unitary similarity of matrices with quadratic minimal polynomials", Linear Algebra Appl., 349:1-3 (2002), 11-16.

[4] Ю. А. Альпин, Х. Д. Икрамов, "Об унитарном подобии алгебр, порождаемых парами ортопроекторов", Численные методы и вопросы организачии вычислений. XVIII, Зап. научн. сем. ПОМИ, 323, ПОМИ, СПб., 2005, 5-14.

[5] F. Kittaneh, "On the structure of polynomially normal operators", Bull. Austral. Math. Soc., 30:1 (1984), 11-18.

[6] M. Huhtanen, "Aspects of nonnormality for iterative methods", Linear Algebra Appl., 394:1 (2005), 119-144.

[7] Х. Д. Икрамов, "Каноническая форма Шура унитарно квазидиагонализуемой матрицы", ЖВМ и МФ, 37:12 (1997), 1411-1415.

\section{Х. Д. Икрамов}

Поступило

Московский государственный университет

10.09 .2008

им. М. В. Ломоносова

E-mail: ikramov@cs.msu.su 